\title{
Motor Evoked Potentials and Bispectral Index-Guided Anaesthesia in Image-Guided Mini-Invasive Neurosurgery of Supratentorial Tumors Nearby the Cortico-Spinal Tract
}

\author{
Roberto CORDELLA ${ }^{1}$, Eleonora ORENA ${ }^{2}$, Francesco ACERBI ${ }^{1}$, Elena BERETTA ${ }^{1}$, Dario CALDIROLI ${ }^{2}$, \\ Francesco DIMECO ${ }^{1}$, Carla CAROZZI ${ }^{2}$
}

${ }^{1}$ Fondazione IRCCS Istituto Neurologico C. Besta, Department of Neurosurgery, Milan, Italy

${ }^{2}$ Fondazione IRCCS Istituto Neurologico C. Besta, Department of Anaesthesia, Milan, Italy

\section{ABSTRACT}

AIM: To describe and evaluate the anaesthesiological regimen used in neurophysiologically monitored image-guided mini-invasive neurosurgery.

MATERIAL and METHODS: Twenty-four patients underwent elective surgery under general anaesthesia that was administered through Target Controlled Infusion (TCl) for effect-site concentration (Ce) of Propofol and Remifentanil, targeting the Bispectral Index (BIS) in the 40-60 intervals. The stimulating intensity of transcranial motor evoked potentials (tMEP), BIS, Propofol and Remifentanil Ce were collected at MEPs threshold (T) definition (respectively BIS@T, CeProp@T and CeRemi@T). Intraoperative seizure, explicit recall for intraoperative awareness and clinical motor status were assessed.

RESULTS: CeProp@T and CeRemi@T ranged respectively between 1.5-2.2 $\mu \mathrm{g} / \mathrm{ml}$ (Median $1.6 \mu \mathrm{g} / \mathrm{ml}$ ) and 3.5-18 ng/ml (Median $8 \mathrm{ng} / \mathrm{ml}$ ) that were effective in keeping the BIS@T between 40 and 60 in all surgeries. tMEP thresholds ranged between 45 and 120 mA. There was no correlation between CeProp@T, CeRemi@T and tMEP, as well as between BIS@T and respectively tMEP, CeProp@T, CeRemi@T. None of patients had induced electrical seizure or explicit recalls. Motor scores were equal to preoperative values in $22 / 24$ patients.

CONCLUSION: BIS-guided general anaesthesia within a 40-60 interval, with low Ce of Propofol ( $\leq 2 \mu / \mathrm{ml})$ and high analgesic regime allow reliable tMEP measurements, avoiding postoperative neurological impairment and major adverse outcomes, such as seizure and awareness.

KEYWORDS: Bispectral index, Brain motor area, Motor evoked potentials, Propofol, Remifentanil

\section{INTRODUCTION}

I n image-guided mini-invasive neurosurgery of supratentorial tumors near the cortico-spinal tract, the pathological tissue is targeted to minimize the trauma to the surrounding healthy nervous system and other functional structures (33). Thus, the motor cortex might not be exposed and its recognition through standard electrocortical stimulation not performed (8). The intraoperative neurophysiological monitor- ing (IOM) approach to mapping and monitoring the corticospinal tract in image-guided mini-invasive neurosurgery was previously described (8). In the above-mentioned approach, the motor-evoked potentials (MEPs) play a pivotal role, and the successful performance of MEP monitoring relies on the careful maintenance of a steady and consistent electrophysiological baseline. Nevertheless, several anaesthetics agents might affect MEPs thresholds (44). 
Bispectral Index (BIS), a depth of anaesthesia (DOA) monitor, might provide an electroencephalography (EEG) correlation with human behavior in the course of general anaesthesia surgeries. BIS value specifies the likelihood of consciousness, thus aiding the tailoring of anaesthetic dosages to the individual patient $(19,34)$. It is recommended to maintain the BIS range between 40 and 60 in general anaesthesia procedures to help avoid inadequate or excessively deep levels of anaesthesia $(13,15,25,40,43)$. The BIS effectiveness in titrating Propofol sedation in patients with frontal lobe tumors has been recently described (36). Nevertheless, scant data are present about BIS guided-anaesthesia in supratentorial neurophysiologically monitored surgeries performed under general or awake anaesthesia $(7,15)$.

This observational study describes the effect of the BIS-guided anaesthesia on MEP thresholds through Target Controlled Infusion (TCl) of Propofol and Remifentanil in image-guided, mini-invasive neurosurgery in tumor removal surgeries near the corticospinal tract. The hypothesis is that the Propofol and Remifentanil dosages do not affect: a) the stimulating threshold for transcranial MEPs (tMEP), b) the BIS index at MEPs threshold.

\section{MATERIAL and METHODS}

\section{Patients}

The study enrolled patients who underwent elective tailored image-guided mini-invasive surgery under Propofol general anaesthesia. The institutional review board approved the study and the patients gave written informed consent prior to surgery. Inclusion criteria to perform an image-guided mini-invasive approach were already described (8). Exclusion criteria to perform $\mathrm{TCl}$ BIS-guided anaesthesia were: patients with a comprehension problem, American Society of Anaesthesiologists score (ASA) >III, Age $<18$ years, body mass index $(\mathrm{BMI})>35$, and $\mathrm{BIS}$ awake $<90$.

Age, gender, ASA status, pre-operative symptoms, antiepileptic drugs (AEDs), tumor location and histological diagnosis were collected. Clinical motor status was assessed pre- and postoperatively ( 1 week and 3 months follow-ups) according to a standard protocol and muscle strength was graded as 0-V (0: no movements; I-II: severe paresis; III-IV: moderate paresis; V: normality) with the British Research Medical Council Scale (BMRC) (21).

Explicit recalls for intraoperative awareness were assessed using the Structured Brice Interview (4) on three postoperative interviews up to 30 days after surgery.

\section{Anaesthesia Protocol and Bispectral Index}

General anaesthesia, from induction to recovery, was administered intravenously through the TCl delivery system (Injectomat ${ }^{\circledR}$ TIVA Agila, Fresenius Kabi, France). TCI pumps incorporated a pharmacokinetic model for the distribution and elimination of anaesthesia drugs from the body. The chosen models were Schnider (39) for Propofol and Minto (23) for Remifentanil. The software pump, depending on the pharmacokinetic model, calculated the necessary drug infusion rate to achieve and maintain the chosen effect-site concentration $(\mathrm{Ce})$ at any time, avoiding overshoot or underdosage during dose adjustments and drug accumulation. The result was a more predictable and stable drug concentration at the brain level $(28,29)$. DOA was unceasingly assessed through the Bispectral Index monitor (BIS Vista, Covidien, USA) during all anaesthesia phases.

Upon arrival at the operating theatre, the 4-contact BIS electrode was positioned on the patient's forehead, contralateral to the lesion. The sensor recorded the raw frontal cortical EEG and EMG. Both signals were digitally converted, through the proprietary BIS algorithm, into alphanumeric values, where 100 represents the awake state and 0 absence of brain electrical activity. Since awareness is generally observed at BIS values higher than 60, while anaesthetic overdose is observed below 40 BIS values, anaesthetics delivery was targeted to keep BIS values between 40 and 60 . A "smoothing time rate" of 15 seconds was selected on the monitor for a faster BIS analysis.

Anaesthesia was induced through a stepwise approach: Propofol infusion started from a target effect-site drug concentration (Cet) of $1.50 \mathrm{mg}^{\mathrm{m}} \mathrm{m}^{-1}$ and was increased by 0.50 mg.ml ${ }^{1}$ steps when balance between $\mathrm{Ce}$, plasma concentration (Cp) and Cet was reached and after at least 1 minute for equilibration, until appearance of loss of consciousness (LOC). This was clinically evaluated as loss of response to mild prodding, according to the Observer Assessment of Alertness/Sedation Scale (OAA/S)(6). Subsequently, Remifentanil infusion was started, its $\mathrm{Ce}$ was increased and hypnotic dosages were decreased, according to the opioid secondary effect. Propofol Ce was reduced in order to keep the BIS value within $40-60$ all through the surgical procedure and the painful IOM sessions. Non-depolarizing muscle relaxants were administered for intubation purposes only, and were not used during the entire surgical session.

Electrical disturbances, $t$ MEP stimulation, artefacts from the surgical field and frontal muscles activation altered the EEG trace, leading to sudden, frequent and persistent increase of BIS value above 60 . To avoid unnecessary overdose of hypnotics, the anaesthetist continuously evaluated the stability of the clinical conditions (blood pressure, heart rate and body movement), as well as the absence of changes on the raw EEG display. If the raw EEG still showed a delta rhythm plus spindle-like waves with absence of fast waves, features of general anaesthesia (2), $\mathrm{TCl}$ infusion was not modified.

BIS value at the $t$ MEP threshold (BIS@T) was recorded at least 15 seconds (in compliance with the selected "smoothing time rate") after completion of each stimulation session, with signal quality index (SQI) $\geq 80 \%$ and EMG signal $\leq 35 \mathrm{db}$, stable clinical conditions, and the EEG delta/spindle-like rhythm was preserved.

\section{Surgical Methodology and Intraoperative MEP Recordings}

Surgical and IOM settings were previously described (8). Briefly, patients were positioned supine on the surgical table with their head elevated above the level of the heart. The image-guided mini-invasive approach included a linear skin 
incision and a small craniotomy centred on the pre-planned trajectory to the tumor (Figure 1). Following dural opening, the 4-contact strip electrode (PMT Corporation, MN, USA) was carefully introduced under the dura towards the motor strip and positioned onto the targeted area according to neuronavigation and electrophysiological data (Figure 1). Tumor removal was achieved under neuronavigation image guidance.

Multimodal neurophysiological monitoring comprised EEG, electrocorticography (ECoG), electromyography (EMG), somatosensory evoked potentials (SSEP) (N20 phase inversion), tMEP and subcortical monopolar stimulation. Nevertheless, for the purpose of this study, only tMEP thresholds were considered for further analyses. Stimulating "corkscrew" electrodes were placed on the scalp at C3/C4 of the International 10-20 System (approximately $7-7.5 \mathrm{~cm}$ lateral to the midline on the central sulcus line). The anode was on the operated side and the cathode was on the contralateral side. Stimulation was performed by a short train of 5 stimuli consisting of rectangular pulses with $1.0 \mathrm{~ms}$ pulse width, and a 30 to $3000 \mathrm{~Hz}$ band-pass filter. The orbicularis oris, deltoid, wrist extensor, abductor pollicis brevis (APB), abductor digiti minimi (ADM), rectus femori, tibialis anterior and extensor digitorum brevi were the muscles selected to monitor the contralateral hemisoma. tMEP thresholds were defined as the lowest electrical current intensity to evoke a muscular response of at least $100 \mathrm{uV}$ in upper limb muscles. Warning criteria were a $50 \%$ decrease in amplitude in more than three consecutive responses, as well as an increase in stimulation amplitude above $20 \%(26,27)$.

\section{Variables Analysed and Statistical Analysis}

The following variables were sampled in all surgical procedures and were considered for analyses: i) Propofol dosages (CeProp@T) and ii) Remifentanil dosages (CeRemi@T), both administered during the tMEP electrical threshold definition and kept throughout sedation; iii) stimulation threshold for tMEP; iv) BIS index observed at the time of tMEP threshold definition (BIS@stimT).

All data were tested for normal distribution by means of the Kolmogorov-Smirnov test (K-S test). According to the K-S test outcomes, either parametric (Pearson product-moment correlation coefficient) or non-parametric (Spearman's rank correlation coefficient) tests were used to evaluate the correlation between i) CeProp@T and stimulation thresholds for tMEP; ii) CeRemi@T and stimulation thresholds for tMEP; iii) BIS@stimT and CeProp@T; iv) BIS@stimT and CeRemi@T; and v) BIS@stimT and stimulation thresholds for tMEP.

The hypothesis was the independency between i) Propofol and Remifentanil dosages and tMEP stimulation threshold; ii) Propofol and Remifentanil dosages and BIS@stimT; and iii) BIS@stimT and stimulation thresholds for tMEP.

\section{RESULTS}

\section{Patients}

Image-guided mini-invasive surgery under Propofol general anaesthesia was performed in 24 patients with ASA status between 1 and 3 (Table I). Fifteen patients were male and 9

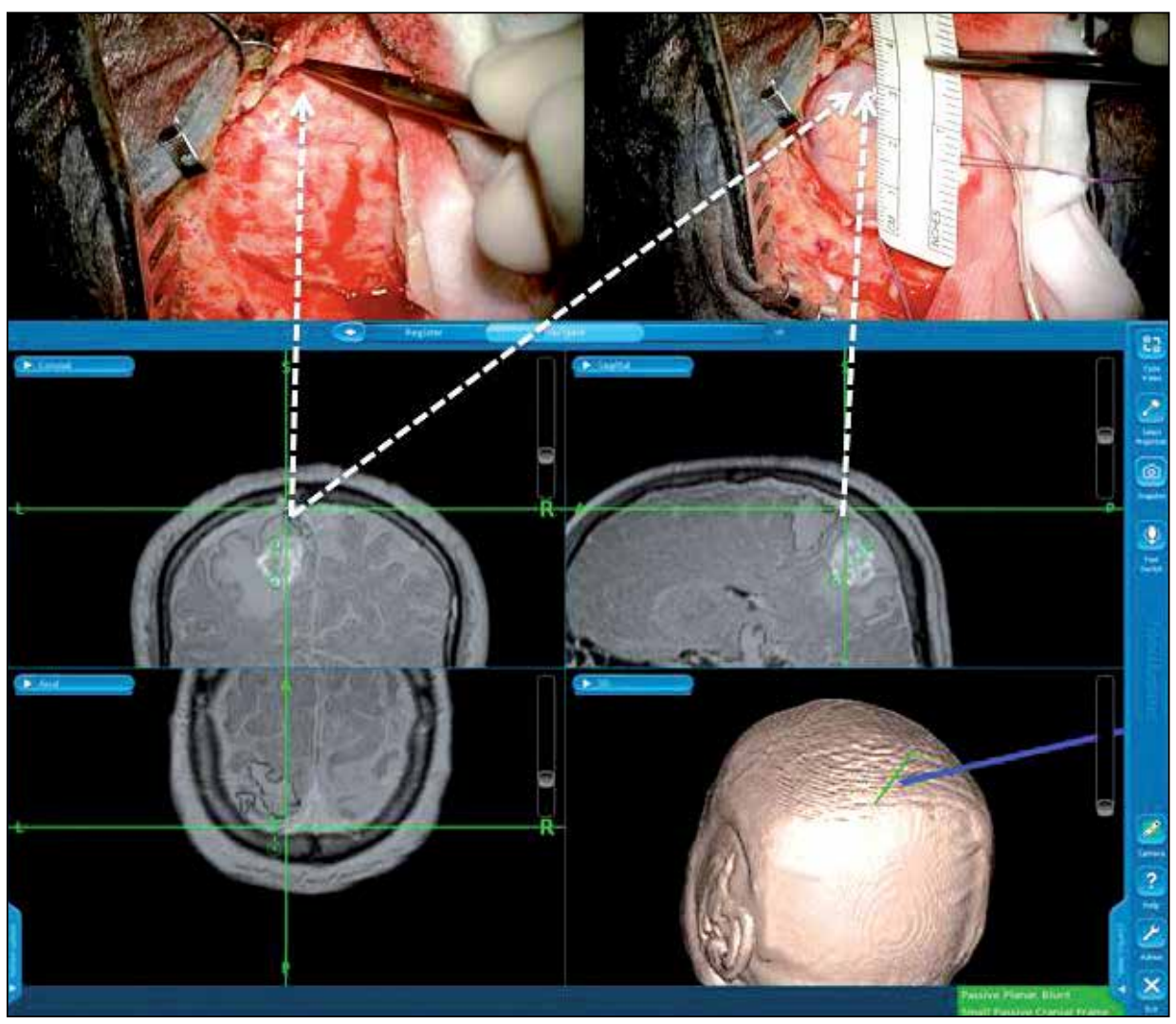

Figure 1: Neuronavigated threedimensional anatomic MRI reconstruction (lower panel) displaying the preoperative planned trajectory (left upper panel) and the superimposed fMRI where the "active" site has guided the 4-contacts strip electrode placement (right upper panel). 
female, with a mean age at surgery of $54 \pm 14$ years (range 30-77 years). Pre-op symptomatology comprised lower limb paresis (9 patients), seizures (6 patients), seizures and lower limb paresis (1 patient), seizures and speech arrest (1 patient), aphasia and upper limb paresis (1 patient), upper limb paresis (1 patient), headache plus upper and lower limb paresis (1 patient), paraesthesia and speech arrest (1 patient), speech arrest (1 patient), mood disorder (1 patient) and loss of consciousness ( 1 patient). Fourteen patients were under antiepileptic drugs (AEDs) either to control or prevent seizures. Nine had levetiracetam, 2 valproic acid, 1 phenobarbital, 1 buxamina, and 1 had a combination of levetiracetam and carbamazepine. Twelve lesions were located in the frontal cortex, 9 were fronto-parietal, 2 were in the gyrus cinguli and 1 was in the fronto-insular region. Fourteen were in the right hemisphere. Histological diagnosis was glioblastoma in 10 patients (World Health Organization-WHO-grade IV), anaplastic astrocytoma in 5 (WHO grade III), anaplastic oligoastrocytoma in 2 (WHO grade III), oligoastrocytoma in 1 (WHO grade III), pilocytic astrocytoma in 1 (WHO grade I), metastatic tumors in 4, and cavernous hemangioma in 1.

\section{Clinical Assessment}

Preoperative BMRC scores were distributed as follow: eleven patients had 5 , nine had 4 ; one had 3 , one had 2 and two had 1.

Postoperative clinical scores were unchanged in 19 patients at the first follow-up (1 week). BMRC scores were equal to those before surgery in 22 out of 24 patients at 3 months follow-up.

Table I: Anaesthesiological and IOM Patients' Data

\begin{tabular}{|c|c|c|c|c|c|c|c|}
\hline Pt ID & $\begin{array}{l}\text { ASA } \\
\text { score }\end{array}$ & $\begin{array}{c}\text { HR } \\
\text { (beats per minute) }\end{array}$ & $\begin{array}{c}\mathrm{BP} \\
(\mathrm{mmHg})\end{array}$ & $\begin{array}{c}\text { Sat } \\
\text { (SpO2) }\end{array}$ & $\begin{array}{c}\mathrm{ETCO}_{2} \\
(\mathrm{mmHg})\end{array}$ & $\begin{array}{c}\text { RR } \\
\text { (breaths per minute) }\end{array}$ & $\begin{array}{l}\mathrm{eV} \\
(\mathrm{ml})\end{array}$ \\
\hline 1 & 2 & 60 & $90 / 60$ & 100 & 27 & 12 & 500 \\
\hline 2 & 2 & 57 & $80 / 50$ & 99 & 27 & 10 & 500 \\
\hline 3 & 2 & 70 & $100 / 60$ & 100 & 28 & 12 & 500 \\
\hline 4 & 2 & 78 & $75 / 50$ & 99 & 28 & 12 & 500 \\
\hline 5 & 2 & 55 & $95 / 60$ & 98 & 29 & 12 & 600 \\
\hline 6 & 2 & 40 & $90 / 60$ & 96 & 32 & 12 & 600 \\
\hline 7 & 2 & 68 & $90 / 60$ & 100 & 31 & 12 & 500 \\
\hline 8 & 2 & 55 & $90 / 50$ & 100 & 25 & 12 & 450 \\
\hline 9 & 2 & 45 & $90 / 60$ & 98 & 31 & 12 & 520 \\
\hline 10 & 2 & 48 & $105 / 70$ & 100 & 27 & 13 & 600 \\
\hline 11 & 2 & 60 & $90 / 60$ & 100 & 30 & 12 & 500 \\
\hline 12 & 2 & 52 & $91 / 61$ & 98 & 27 & 10 & 540 \\
\hline 13 & 2 & 45 & $110 / 75$ & 99 & 27 & 12 & 600 \\
\hline 14 & 3 & 45 & $100 / 60$ & 97 & 28 & 12 & 600 \\
\hline 15 & 1 & 47 & $100 / 45$ & 99 & 29 & 12 & 700 \\
\hline 16 & 2 & 68 & $110 / 65$ & 100 & 29 & 12 & 600 \\
\hline 17 & 2 & 45 & $95 / 60$ & 100 & 32 & 12 & 600 \\
\hline 18 & 1 & 55 & $90 / 60$ & 100 & 34 & 11 & 600 \\
\hline 19 & 2 & 46 & $90 / 50$ & 98 & 30 & 12 & 600 \\
\hline 20 & 2 & 45 & $90 / 60$ & 100 & 29 & 12 & 550 \\
\hline 21 & 2 & 54 & $90 / 60$ & 97 & 31 & 12 & 660 \\
\hline 22 & 2 & 46 & $95 / 70$ & 99 & 33 & 12 & 600 \\
\hline 23 & 2 & 51 & $104 / 75$ & 100 & 28 & 12 & 550 \\
\hline 24 & 1 & 81 & $95 / 58$ & 99 & 26 & 12 & 500 \\
\hline
\end{tabular}

ASA: American Society of Anaesthesiology, BP: Blood pressure, ETCO${ }_{2}$ End-tidal carbon dioxide tension, HR: Heart rate, Sat: Oxygen saturation, RR: Respiratory rate, eV: Expiratory Volume. 
Only 2 patients showed a score worsening, with specifically one regressing from 5 to 4 and the other from 4 to 3 .

None of the patients reported explicit memory of intraoperative events at the postoperative interview (1 week), or at the second follow-up (1 month).

\section{Anaesthesiological Management}

$\mathrm{TCl}$ effect compartment concentration $(\mathrm{Ce})$ at the stimulation threshold definition ranged between $1.5-2 \mathrm{ng} / \mathrm{ml}$ (Median 1.6 $\mathrm{mg} / \mathrm{ml}$ ) for Propofol and 3.5-18 $\mathrm{ng} / \mathrm{ml}$ (Median $8 \mathrm{ng} / \mathrm{ml}$ ) for Remifentanil (Table II). These dosages allowed keeping BIS values in the 40 to 60 range (See example in Figure 2) during all surgical procedures (Table II).

Aiming to standardize the action of carbon dioxide on the cerebral blood flow, the end-tidal carbon dioxide tension $\left(\mathrm{ETCO}_{2}\right.$ ) constantly ranged between 25 and $33 \mathrm{~mm} \mathrm{Hg}$, with tidal volumes between 6 to $10 \mathrm{ml} / \mathrm{kg}$ during mechanical ventilation for the duration of the procedure (Table I). Blood pressure (BP), heart rate (HR), oxygen saturation (Sat), respiratory rate $(\mathrm{RR})$ and expiratory volume $(\mathrm{eV})$ are reported in Table I.

\section{tMEP}

tMEPs of at least $100 \mu \mathrm{V}$ were recorded with stimulation thresholds ranging between 45 and $120 \mathrm{~mA}$ (Table II). Across all the surgical procedures, 1) potentials were stable and replicable; 2) no $50 \%$ decrease in amplitude nor a higher than $20 \%$ increase in stimulation threshold were recorded; 3) no electrical induced seizures were documented.

\section{Statistical Analysis}

All data sets passed the Kolmogorov-Smirnov (K-S) test, and indeed the parametric test Pearson product-moment correlation coefficient was applied. No statistical significance resulted between the following: i) CeProp@T and stimulation threshold for tMEP ( $r=-0.1505, p=0.4827$; Table II), ii) CeRemi@T and stimulation threshold for tMEP $(r=0.1941$, $\mathrm{p}=0.3633$; Table II), iii) BIS@stimT and CeProp@T ( $r=-0.1948$, $\mathrm{p}=0.3616$; Table II), iv) BIS@stimT and CeRemi@T ( $r=0.1199$, $\mathrm{p}=0.5769$; Table II), and v) BIS@stimT and tMEP stimulation threshold ( $r=-0.02575, p=0.9049$; Table II).

\section{DISCUSSION}

This observational study draws attention to the effect of the BIS-guided anaesthesia on $t$ MEPs thresholds in imageguided mini-invasive neurosurgery. BIS has guided the drugs titration, thus allowing low doses of Propofol $(\leq 2 \mathrm{mg} / \mathrm{ml})$ and high doses of opioids to minimize the impact of anaesthetics on $t$ MEPs. Our data point to a lack of correlation between Propofol dosages, Remifentanil dosages, BIS index and $t$ MEP stimulation thresholds.

BIS is a non-invasive technology developed to evaluate the level of sedation on the principle that chances in the EEG waveforms can affect the level of awareness. EEG shows high frequency and low amplitude waveforms during wakefulness (3). On the other hand, the frequency is low and the amplitude is high during deep sedation (34). The BIS index is an algorithm for digital signal processing developed from the above-mentioned EEG principles (41). More precisely, it is an alphanumeric index ranging from 0 to 100 that well correlates with the level of sedations (13). An index ranging from 70 to 90 corresponds to light to moderate sedation, 60-70 to deep sedation, 40-60 to general anaesthesia and below 40 to deep hypnotic state.

BIS ranging from 40 to 60 is targeted because the detection of muscular response is reported to be challenging at values below 40, and higher electrical stimulation intensities might be required to evoke MEP, which leads to an increased likelihood of seizures (45). Conversely, with an index above 60, a higher probability of intraoperative recalls has been reported (20). Interestingly, in the reported series, electrical stimulation

Table II: Anaesthesiological Regimen and tMEP Thresholds

\begin{tabular}{|c|c|c|c|c|}
\hline Pt ID & $\begin{array}{c}\text { Ce Propofol } \\
(\mu g)\end{array}$ & $\begin{array}{c}\text { Ce Ultiva } \\
\text { (ng) }\end{array}$ & $\begin{array}{r}t \text { MEP } \\
(\mathrm{mA})\end{array}$ & BIS@Stim \\
\hline 1 & 1.5 & 12 & 120 & 46 \\
\hline 2 & 1.7 & 6.0 & 100 & 47 \\
\hline 3 & 1.6 & 8.5 & 100 & 47 \\
\hline 4 & 1.9 & 8.0 & 105 & 48 \\
\hline 5 & 1.6 & 8.0 & 115 & 48 \\
\hline 6 & 1.7 & 7.0 & 105 & 50 \\
\hline 7 & 1.5 & 10 & 80 & 53 \\
\hline 8 & 1.5 & 12.1 & 70 & 47 \\
\hline 9 & 2.2 & 8.0 & 75 & 45 \\
\hline 10 & 1.8 & 18 & 85 & 51 \\
\hline 11 & 1.5 & 10 & 80 & 45 \\
\hline 12 & 1.7 & 6.1 & 100 & 50 \\
\hline 13 & 1.5 & 3.5 & 70 & 50 \\
\hline 14 & 1.5 & 3.5 & 85 & 45 \\
\hline 15 & 1.4 & 13 & 100 & 45 \\
\hline 16 & 2.0 & 8.7 & 75 & 48 \\
\hline 17 & 2.0 & 12.0 & 75 & 50 \\
\hline 18 & 1.6 & 11.0 & 65 & 45 \\
\hline 19 & 1.8 & 6.0 & 50 & 53 \\
\hline 20 & 1.5 & 5.5 & 45 & 51 \\
\hline 21 & 1.5 & 12.0 & 50 & 48 \\
\hline 22 & 1.5 & 5.0 & 60 & 51 \\
\hline 23 & 1.8 & 6.0 & 65 & 45 \\
\hline 24 & 2.2 & 3.0 & 60 & 48 \\
\hline
\end{tabular}


induced seizures were not documented and none of the patients experienced explicit intraoperative recalls at either follow-up.

Propofol has been chosen as it produces a more reliable neurophysiological environment for monitoring than inhalational anaesthetics $(24,31,32,44)$. Specifically, Propofol anaesthesia has a milder effect on MEP than the latter (35). The reported data suggest that Propofol and Remifentanil dosages had a low probability of correlating both with $t$ MEP thresholds and with the BIS number associated with $t$ MEP thresholds. It may indeed be speculated that the interaction of both drugs, not the single agent, modulated the BIS. Also, the 40 to $60 \mathrm{BIS}$ range did not interfere with MEP monitoring. Actually, it has been reported that during Propofol anaesthesia, high-dose Remifentanil enhances the hypnotic effect of Propofol, and consequently its concentration might be markedly reduced (22). Furthermore, opioids produce minimal or no EEG variation, although when added to hypnotic agents, the BIS range decreases as a consequence of the reduced Propofol dosage (42). This is the so-called "opioid secondary effect" (10), allowing to safely reduce Propofol dosages, thus keeping a 40 to $60 \mathrm{BIS}$ range. This methodology is of great importance to IOM, as our data indicate how low an impact anaesthesiology conditions have had on MEPs thresholds. Specifically, no changes in the electrical thresholds during tumour removal have been recorded, which is paramount as there is no need to increase stimulation intensities, as a result of the stable amplitude of potentials compared to the baseline. It is worthwhile mentioning that in our setting the electrical threshold has been defined as the lowest intensity evoking a muscular potential of at least $100 \mu \mathrm{V}$ in upper limb muscles. Therefore, a threshold increase would have elicited physiological changes due to the state of surgery (5), rather than to the subjective changes in Propofol concentrations. It is our opinion that this conduct lessens the impact of the anaesthesia regimen as a possible confounding factor when electrophysiological changes are detected during the surgery, thus motivating the neurophysiologist to promptly communicate variations to the surgeons. The administration of low-dose Propofol during the surgical procedures should be emphasized, as strictly connected with MEP amplitude. Kajiyama et al.(16) suggested that when transcranial MEP is monitored under Propofol anaesthesia, controlling anaesthetic conditions for reliable measurements is paramount. Nathan et al.(24) demonstrated that a Propofol concentration ranging from 4 to $8 \mathrm{mg} / \mathrm{l}$ reduces MEP amplitude, with no effect on latencies. Furthermore, motor neuron excitability is markedly impaired when the target Propofol concentration reaches 9 $\mathrm{mg} / \mathrm{l}$ (18). Kakinohana et al.(17) illustrated how, owing to the inhibition of spinal motor neuron excitability, transcranial MEP evoked by motor cortex single-shock stimulation may not be well preserved at a Propofol concentration larger than $2 \mu \mathrm{g} / \mathrm{ml}$. In the described series, Propofol concentration was consistently lower, ranging between 1.5 and $2 \mu \mathrm{g} / \mathrm{ml}$. Intravenous anaesthetics have an inhibitory effect on the cortical axon synapses and spinal anterior horn cells, and show a tendency to depress motor neuron activity. Propofol

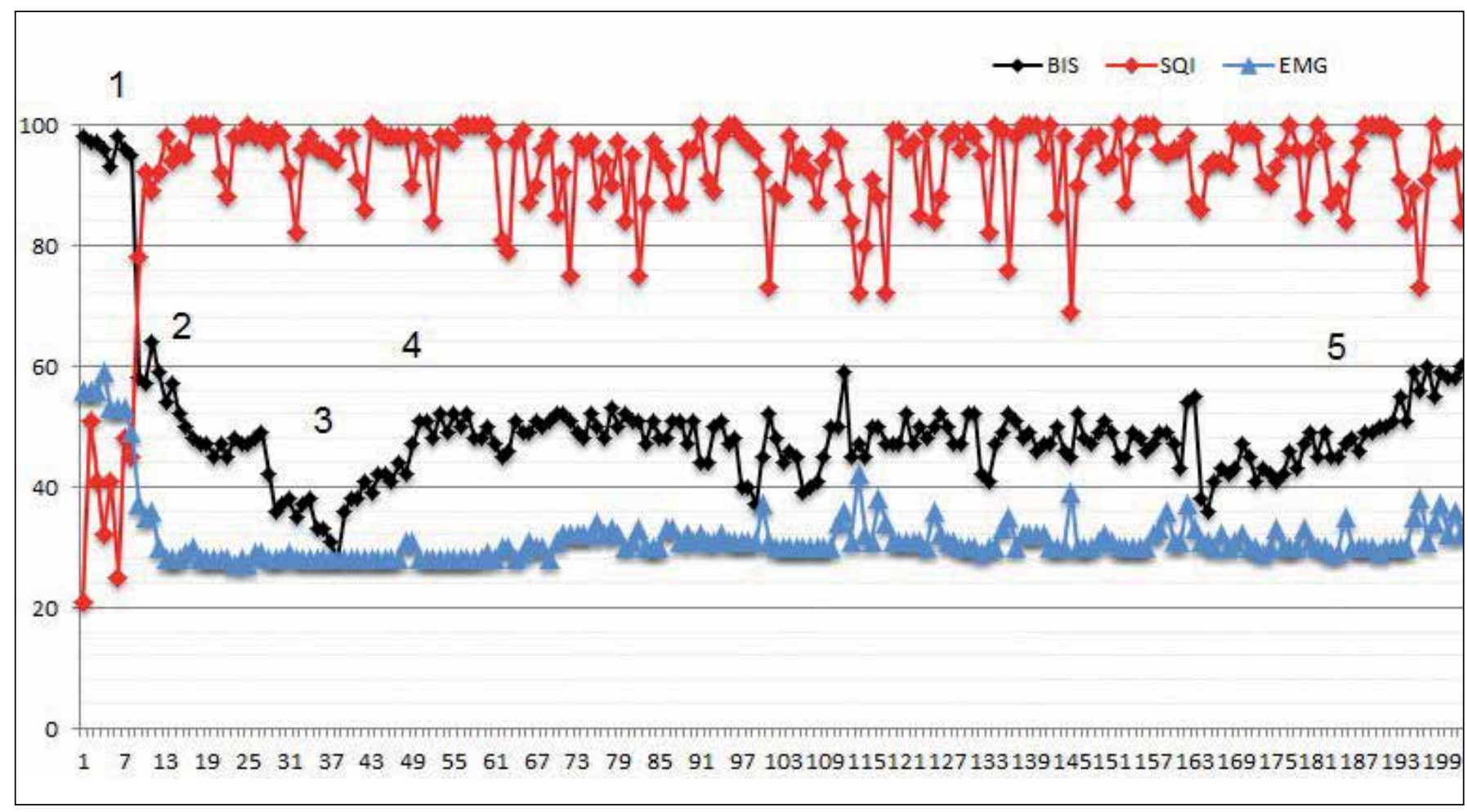

Figure 2: Illustrative case of BIS trend during surgery. Numbers point out to specific events during the procedures: 1-placing the BIS sensor and beginning the sedation; 2-intubation; 3-epidural strip's placement; 4-stimulation from strip before its removal; 5-haemostasis. The $\left.{ }^{*}\right)$ symbol labels artifacts on BIS values due to tMEP stimulation. 
potentiates aminobutyric acid type A receptor channels (30), and this can depress spinal motor neuron excitability. Guertin and Hounsgaard (14) validated that Propofol reduces the spinal motor neurons excitability by suppressing the plateau potentials mediated by L-type calcium channels.

On the other hand, opioids like Remifentanil have been shown to play a minimal role in MEP recording. Similarly to Propofol, such opioids suppress myogenic potentials in a dose-dependent fashion, although this effect on Compound Muscle Action Potentials (CMAP) amplitudes is rather moderate compared to Propofol, and, remarkably, all the other opioids investigated (38). MEPs are indeed not abolished, even at high Remifentanil infusion rates (15 ng/ $\mathrm{ml})$. Due to their short context-sensitive half-life, Propofol and Remifentanil are well suited for neurosurgical procedures, in which early postoperative emergence from anaesthesia is desirable. The described anaesthesiological actions, along with the peculiar IOM setting planned in an image-guided mini-invasive neurosurgical approach, also yield encouraging clinical effects. Only $8 \%$ of the patients $(n=2)$ reported a mild worsening of the motor functions at three-month follow-up, which is comparable to incidences reported in larger series operated through standard approach $(1,9,11,12,37)$.

There are some limitations of this study that need to be documented. The population of the study is small and focusing on patients undergoing a minimally invasive approach in a limited period of time, requiring future studies to be planned with data sampled from more patients. The administration of low-dose Propofol and the likelihood of intraoperative awareness may also come under fire, although, as previously stated, none of our patients described explicit intraoperative recalls. This, however, might be due to the small sample size. More thorough and focused studies are therefore required to shed further light on this feature. Intraoperative awareness may also increase the probability of intraoperative implicit memories, which include insomnia, depression, and anxiety-like symptoms that have not been systematically investigated, although none of the patients reported to have experienced any of the above symptoms at three-month phone interviews. It might accordingly be of pertinence to include this potential feature of intraoperative awareness in future studies. Furthermore, the image-guided minimally invasive approach is not applicable to all surgical procedures involving eloquent areas, whereas awake craniotomy and electrocortical stimulation are still the gold standard (1). Indeed, tumor characteristics - such as the diameter of the lesion, which should be less than $4 \mathrm{~cm}$, and the proximity to the cortico-spinal tract and its position, limited the possibility to perform small craniotomies and imageguided trajectories. Nevertheless, the aim of this report was to investigate the effectiveness of this anaesthesia-IOM protocol, evaluating firstly its practicability with particular attention to the relationship between tMEPs thresholds and the incidence of intraoperative awareness.

\section{CONCLUSION}

This study describes the anaesthesiological management during image-guided mini-invasive neurosurgical procedures to remove tumors in near the motor cortex. Propofol and opioid dosage was managed to keep the BIS between 40 and 60. Specifically, data suggest that low Propofol doses and high opioid doses can minimize the effect of anaesthesia on electrophysiological monitoring, which, in this kind of surgical procedures, is pivotal to ensure low post-op morbidity and no intraoperative explicit recalls.

\section{- REFERENCES}

1. Bello L, Fava E, Casaceli G, Bertani G, Carrabba G, Papagno C, Falini A, Gaini SM: Intraoperative mapping for tumor resection. Neuroimaging Clin N Am 19: 597-614, 2009

2. Bennett C, Voss LJ, Barnard JP, Sleigh JW: Practical use of the raw electroencephalogram waveform during general anaesthesia: The art and the science. Anesth Analg 109: 539550, 2009

3. Berger H: Über das Elektrenkephalogramm des Menschen. Archiv für Psychiatrie und Nervenkrankheiten 87: 527-570, 1929 (In German)

4. Brice DD, Hetherington RR, Utting JE: A simple study of awareness and dreaming during anaesthesia. $\mathrm{Br} J$ Anaesth 42: 535-542, 1970

5. Calancie B, Harris W, Brindle GF, Green BA, Landy HJ: Threshold-level repetitive transcranial electrical stimulation for intraoperative monitoring of central motor conduction. J Neurosurg 95 Suppl 2:161-168, 2001

6. Chernik D, Gillings D, Laine, H, Hendler J, Silver JM, Davidson $A B$, Schwam EM, Siegel JL: Validity and reliability of the Observer's Assessment of Alertness/Sedation Scale: Study with intravenous midazolam. J Clin Psychopharmacol 10(4): 244-251, 1990

7. Conte V, L'acqua C, Rotelli S, Stocchetti N: Bispectral index during asleep-awake craniotomies. J Neurosurg Anesthesiol 25: 279-284, 2013

8. Cordella R, Acerbi F, Broggi M, Vailati D, Nazzi V, Schiariti M Tringali G, Ferroli P, Franzini A, Broggi G: Intraoperative neurophysiological monitoring of the cortico-spinal tract in image-guided mini-invasive neurosurgery. Clin Neurophysiol 124: 1244-1254, 2013

9. Cordella R, Acerbi F, Marras CE, Carozzi C, Vailati D, Saini M, Tringali G, Ferroli P, Dimeco F, Franzini A, Broggi G: Risk of seizures during intraoperative electrocortical stimulation of brain motor areas: A retrospective study on 50 patients. Neurol Sci 34(1):63-70, 2013

10. Dahaba AA: Different conditions that could result in the Bispectral index indicating an incorrect hypnotic state. Anesth Analg 101:765-773, 2005

11. Duffau H: Contribution of cortical and subcortical electrostimulation in brain glioma surgery: Methodological and functional considerations. Neurophysiol Clin 37: 373-382, 2007

12. Duffau H, Capelle L, Sichez JP, Faillot T, Abdennour L, Law Koune JD, Dadoun S, Bitar A, Arthuis F, Van Effenterre R, Fohanno D: Intraoperative direct electrical stimulations of the central nervous system: The Salpetriere experience with 60 patients. Acta Neurochir (Wien) 141:1157-1167, 1999

13. Glass PS, Bloom M, Kearse L, Rosow C, Sebel P, Manberg $P$ : Bispectral analysis measures sedation and memory effects of propofol, midazolam, isoflurane and alfentanil in healthy volunteers. Anesthesiology 86:836-847, 1997 
14. Guertin PA, Hounsgaard J: Non-volatile general anaesthetics reduce spinal activity by suppressing plateau potentials. Neuroscience 88: 353-358, 1999

15. Hans P, Bonhomme V, Born JD, Maertens de Noordhoudt A, Brichant JF, Dewandre PY: Target-controlled infusion of propofol and remifentanil combined with bispectral index monitoring for awake craniotomy. Anaesthesia 55:255-259, 2000

16. Kajiyama S, Sanuki M, Kinoshita H: Effect of bolus propofol administration on muscle evoked potential (MsEP) during spine surgery. Masui 50:867-873, 2001

17. Kakinohana M, Fuchigami T, Nakamura S, Kawabata T, Sugahara K: Propofol reduces spinal motor neuron excitability in humans. Anesth Analg 94:1586-1588, 2002

18. Kerz T, Hennes HJ, Feve A, Decq P, Filipetti P, Duvaldestin P: Effects of propofol on H-reflex in humans. Anesthesiology 94 : 32-37, 2001

19. Kissin I: Depth of anesthesia and bispectral index monitoring. Anesth Analg 90:1114-1117, 2000

20. Liu J, Singh H, White PF: Electroencephalographic bispectral index correlates with intraoperative recall and depth of propofol-induced sedation. Anesth Analg 84:185-189, 1997

21. Medical Research Council: Aids to the examination of the peripheral nervous system, memorandum no. 45. London: Her Majesty's Stationery Office, 1981

22. Milne SE, Kenny GN, Schraag S: Propofol sparing effect of remifentanil using closed-loop anaesthesia. $\mathrm{Br} \mathrm{J}$ Anaesth 90: 623-629, 2003

23. Minto CF, Schnider TW, Shafer SL: Pharmacokinetics and pharmacodynamics of remifentanil. II. Model application. Anesthesiology 86: 24-33, 1997

24. Nathan N, Tabaraud F, Lacroix F, Moulies D, Viviand X, Lansade A, Terrier G, Feiss P: Influence of propofol concentrations on multipulse transcranial motor evoked potentials. $\mathrm{Br} \mathrm{J}$ Anaesth 91: 493-497, 2003

25. National Institute for Health and Care Excellence: Depth of anaesthesia monitors Bispectral index, E-Entropy and Narcotrend-Compact M. www.nice.org, 2012

26. Neuloh G, Pechstein U, Cedzich C, Schramm J: Motor evoked potential monitoring with supratentorial surgery. Neurosurgery 54: 1061-1070, 2004

27. Neuloh G, Schramm J: Motor evoked potential monitoring for the surgery of brain tumors and vascular malformations. Adv Tech Stand Neurosurg 29: 171-228, 2004

28. Nimmo AF, Cook TM: Total intravenous anaesthesia. In: Pandit JJ, Cook TM (eds). Accidental Awareness during General Anaesthesia in the United Kingdom and Ireland. The Royal College of Anaesthetists and the Association of Anaesthetists of Great Britain and Ireland, 2014

29. Nimmo AF, Pandit JJ: Depth of anaesthesia monitoring. In: Pandit JJ, Cook TM (eds). Accidental Awareness during General Anaesthesia in the United Kingdom and Ireland, The Royal College of Anaesthetists and the Association of Anaesthetists of Great Britain and Ireland, 2014

30. Orser BA, McAdam LC, Roder S, MacDonald JF: General anesthetics and their effects on $\operatorname{GABA}(A)$ receptor desensitization. Toxicol Lett 100-101: 217-224, 1998
31. Pechstein U, Nadstawek J, Zentner J, Schramm J: Isoflurane plus nitrous oxide versus propofol for recording of motor evoked potentials after high frequency repetitive electrical stimulation. Electroencephalogr Clin Neurophysiol 108:175181, 1998

32. Pelosi L, Stevenson M, Hobbs GJ, Jardine A, Webb JK: Intraoperative motor evoked potentials to transcranial electrical stimulation during two anaesthetic regimens. Clin Neurophysiol 112: 1076-1087, 2001

33. Perneczky A, Muller-Forell W, van Lindert E, Fries G: Keyhole concept in neurosurgery. Stuttgart, New York: Thieme Medical Publishers, 1999

34. Rampil IJ: A primer for EEG signal processing in anesthesia. Anesthesiology 89: 980-1002, 1998

35. Rohde V, Krombach G A, Baumert JH, KreitschmannAndermahr I, Weinzierl M, Gilsbach JM: Measurement of motor evoked potentials following repetitive magnetic motor cortex stimulation during isoflurane or propofol anaesthesia. Br J Anaesth 91:487-492, 2003

36. Sahinovic HM, Beese U, Heeremans EH, Kalmar A, van Amsterdam K, Steenbakkers RJ, Kuiper H, Spanjersberg R, Groen RJ, Struys MM, Absalom AR: Bispectral index values and propofol concentrations at loss and return of consciousness in patients with frontal brain tumors and control patients. Br J Anaesth 112(1):110-117, 2014

37. Sanai N, Berger MS: Intraoperative stimulation techniques for functional pathway preservation and glioma resection. Neurosurg Focus 28: E1, 2010

38. Scheufler KM, Zentner J: Total intravenous anesthesia for intraoperative monitoring of the motor pathways: An integral view combining clinical and experimental data. J Neurosurg 96: 571-579, 2002

39. Schnider TW, Minto CF, Shafer SL, Gambus PL, Andresen C, Goodale DB, Shafer SL, Youngs EJ: The influence of age on propofol pharmacodynamics. Anesthesiology 90:1502-1516, 1990

40. Sebel PS, Land E, Rampil IJ, White PF, Cork R, Jopling M, Smith NT, Glass PS, Manberg P: A multicentre study of bispectral electroencephalogram analysis for monitoring anesthetic effect. Anesth Analg 84: 891-899, 1997

41. Sigl JC, Chamoun N: An introduction to bispectral analysis for encephalogram. J Clin Monit 10:392-404, 1994

42. Strachan AN, Edwards ND: Randomized placebo-controlled trial to assess the effect of remifentanil and propofol on bispectral index and sedation. $\mathrm{Br} \mathrm{J}$ Anaesth 84: 489-490, 2000

43. Struys M, Versichelen L, Mortier E, Ryckaert D, De Mey JC, De Deyne C, Rolly G: Comparison of spontaneous frontal EMG, EEG power spectrum and bispectral index to monitor propofol drug effect and emergence. Acta Anaesthesiol Scand 43: 94102, 1998

44. Wang AC, Than KD, Etame AB, La Marca F, Park P: Impact of anesthesia on transcranial electric motor evoked potential monitoring during spine surgery: A review of the literature. Neurosurg Focus 27(4): E7, 2009

45. Yamaguchi F, Oi Y, Aoki W, Nakamura R, Igarashi A, Kubota M, Sawada K, Shimura T, Takahashi H, Kobayashi S, Teramoto A: BIS monitoring is useful for reliable intraoperative cortical mapping during brain tumor operations. No Shinkei Geka 30: 1181-1188, 2002 (In Japanese) 